\title{
Right anterior minithoracotomy for aortic valve replacement: 10-year experience of a single center
}

\author{
Mattia Glauber, MD, Daniyar Gilmanov, MD, Pier Andrea Farneti, MD, Enkel Kallushi, MD, \\ Antonio Miceli, MD, Francesca Chiaramonti, MD, Michele Murzi, MD, and Marco Solinas, MD
}

\section{ABSTRACT}

Objective: Minimally invasive aortic valve replacement (AVR) has been associated with several better outcomes over the standard full sternotomy approach. We revised our 10-year experience with right anterior minithoracotomy (RAMT) for AVR.

Methods: Between 2004 and 2014, a total of 593 patients (310 men; median age: 73.8 years) underwent AVR via RAMT. Preoperatively, a mixed valve lesion was diagnosed in $55(9.3 \%)$ patients; and pure aortic regurgitation in $86(14.5 \%)$. Mean logistic EuroSCORE I (European system for cardiac operative risk evaluation) was 7.4 (median: 5.76).

Results: In $302(50.9 \%)$ patients, a sutureless or rapidly implantable biological prosthesis was used; in $23(3.9 \%)$, a mechanical prosthesis; and in the remainder, a conventional biological prosthesis. A total of $113(19.1 \%)$ patients had a small aortic annulus $(\leq 21 \mathrm{~mm})$. Operative times averaged 80 (median: 74$)$ minutes of crossclamping time, and 117 (107) minutes of perfusion time; these were significantly shorter with a sutureless prostheses, compared with a sutured prostheses: perfusion 99 versus 134 minutes, $P<.0005$; aortic crossclamping time: 64 versus 97 minutes, $P<.0005$. The mean (median) assisted ventilation time was 9.8 (6) hours; intensive care unit stay was 1.5 (1) days; hospital length of stay was 6.6 (6) days. Overall in-hospital mortality was 9 deaths (1.5\%). At 31.5 months mean follow-up time (1531 cumulative patient-years), 94.8\% survival was observed.

Conclusions: Minimally invasive AVR is a safe procedure, with low perioperative morbidity, and low rates of reoperation and death at late follow-up. Excellent outcomes can be achieved with minimally invasive AVR via right anterior minithoracotomy. Sutureless prostheses facilitate minimally invasive AVR and are associated with reduced operative times. (J Thorac Cardiovasc Surg $2015 ; 150: 548-56)$

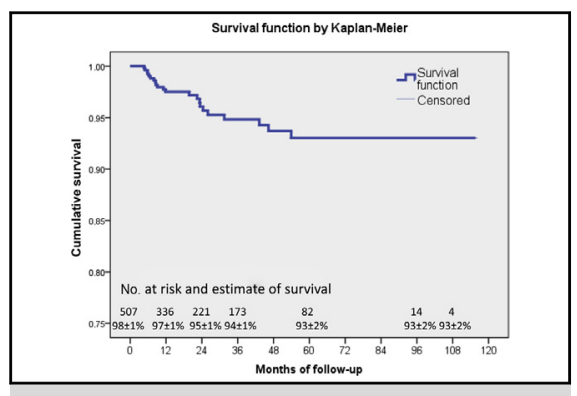

Overall survival curve by Kaplan-Meier test for general cohort (593 patients).

\section{Central Message}

Aortic valve replacement through right anterior minithoracotomy provides excellent outcomes. Sutureless prostheses facilitate mini aortic valve replacement.

\section{Perspective}

Many patients with aortic valve disease still undergo conventional AVR or are directed to alternative treatment with transcatheter AVR. Mini-AVR, through RAMT, provides excellent short-term outcomes, with low mortality and perioperative morbidity, and comparable longterm survival. More surgeons should enrich their armamentarium by adding RAMT AVR. Sutureless prostheses can increase adoption of RAMT AVR.

See Editorial page 450 .
Supplemental material is available online.

To reduce surgical trauma, several minimally invasive approaches (eg, right parasternal approach, upper and lower ministernotomy, V-shaped, inverse-T, J- and reverse-L partial ministernotomy, transverse sternotomy, and right

From the Department of Adult Cardiac Surgery, G. Pasquinucci Heart Hospital, Gabriele Monasterio Foundation, Massa, Italy.

Received for publication Feb 15, 2015; revisions received May 16, 2015; accepted for publication June 5, 2015; available ahead of print July 26, 2015. minithoracotomy) have been developed for aortic valve replacement $^{1-4}$ (AVR) since 1993 and have been associated with better outcomes and lower perioperative morbidity compared with full sternotomy. ${ }^{5-13}$

Partial sternotomy seems to be the most widely adopted option for minimally invasive AVR, ,6,14-17 whereas right anterior minithoracotomy (RAMT) has the potential to offer better outcomes-namely, lower incidence of 


\section{Abbreviations and Acronyms \\ AVR $=$ aortic valve replacement \\ $\mathrm{CABG}=$ coronary artery bypass grafting \\ $\mathrm{CI} \quad=$ confidence interval \\ $\mathrm{CPB}=$ cardiopulmonary bypass \\ EuroSCORE $=$ European system for cardiac operative risk evaluation \\ ICU = intensive care unit \\ IQR $\quad=$ interquartile range \\ NYHA $=$ New York Heart Association \\ OR $=$ odds ratio \\ RAMT = right anterior minithoracotomy}

postoperative atrial fibrillation, and shorter ventilation time, intensive care unit stay, and hospital stay. ${ }^{4,8,9,12,18}$ However, with limited space to work, operative times are generally longer with minimally invasive approaches, $4,8,12,18,19$ although reduced aortic crossclamping times and cardiopulmonary bypass (CPB) times were reported with minimally invasive AVR as well. ${ }^{15}$

To overcome the technical challenges of AVR, and to shorten implantation time, a new generation of aortic valve prostheses have been introduced in clinical practice. ${ }^{20} \mathrm{Su}$ tureless AVR features shorter operative times, and probably, better outcomes compared with conventional surgery. ${ }^{21}$ Few studies have evaluated RAMT for AVR and its remote outcomes. ${ }^{9-12}$ We aimed to analyze our 10-year experience with this approach and to compare early and midterm outcomes after conventional versus sutureless AVR.

\section{METHODS}

The clinical audit committee of the G. Pasquinucci Heart Hospital institutional review board approved the study as meeting ethical and legal requirements. All patients gave written informed consent.

\section{Patient Selection and Enrollment}

A retrospective, observational cohort study was conducted with prospective data collection on 3313 consecutive patients who underwent an aortic valve procedure in our institution between August 2004 and May 2014. Patients, operated on with a right minithoracotomy or a small thoracotomy, were selected, and the final study population consisted of 593 patients. Thirteen patients with conversion to full sternotomy were analyzed as intent-to-treat.

\section{Patient Morbidity and Survival}

Overall mortality was defined as death for any reason, inclusive of events occurring $<30$ days from the procedure. Postoperative morbidity was defined according to information in the Society of Thoracic Surgeons database. Prolonged intensive care unit (ICU) stay was defined as $\geq 3$ days in the ICU, immediately after surgery and afterward. New-onset atrial fibrillation was defined by documentation of atrial fibrillation of any duration at any point in the postoperative period, on a rhythm strip or 12-lead electrocardiogram.

All patients were seen 2 to 3 months after surgery, and thereafter were contacted for follow-up data. Patient survival was determined by telephone calls, e-mail, surface mail, or out-patient interview. The median follow-up period was 31 months (interquartile range [IQR]: 10-49 months; cumulative follow-up: 1531 patient-years); follow-up data were $98 \%$ complete.

\section{Anesthesia, Surgical Technique, and Postoperative Management}

Anesthesia and surgical techniques were standardized according to institutional protocols for all patients, and have been described elsewhere. $^{22,23}$ The choice of a specific, minimally invasive approach for AVR in our institution depends on the suitability of the patient's anatomy for RAMT, which is our first-line approach. The exclusion criteria for RAMT were: previous right-side chest surgery; a history of right pleurisy or previous right-side pleural effusion with adhesion formation; severe chest wall deformities; severe pulmonary bullous disease; and the presence of an ascending aorta aneurysm or the technical impossibility of obtaining peripheral percutaneous venous cannulation.

Furthermore, patients were considered suitable for RAMT if the following computed tomography scan criteria were met: (1) at the level of the main pulmonary artery, the ascending aorta was rightward (more than one-half located on the right in respect to the right sternal border); and (2) the distance from the ascending aorta to the sternum did not exceed $10 \mathrm{~cm}$. However, in the sutureless era (since April 2011), these computed tomography criteria had only relative importance for the choice of surgical approach, and not every patient met them who underwent surgery for AVR through RAMT after May 2011. No specific selection criteria were applied for sutureless prostheses: since they became available, every patient considered for biological prosthesis implantation via RAMT was a potential candidate for sutureless AVR; however, type 1 and 2 bicuspid aortic valves without raphe and asymmetric aortic root were considered unfavorable anatomic conditions for sutureless AVR.

\section{Statistical Analysis}

The composite event score included the following complications: need for mechanical ventilation support for $>24$ hours, reoperation for any cause, perioperative acute myocardial infarction, stroke, transient ischemic attack, new-onset dialysis, multisystem failure, atrioventricular block that required permanent pacemaker implantation, cardiorespiratory arrest, need for percutaneous pericardial drainage, major arrhythmia, and death during the index hospitalization. A multivariable ordinal regression model based on preoperative and intraoperative variables (Appendix E1) was used for composite event score analysis.

Overall survival was evaluated using the Kaplan-Meier method, with an actuarial survival graph.

Factors found to trend toward significance by univariate testing $(P<.10)$ were entered into a multivariable analysis (a total of 110 variables evaluated; Appendix E1). Binary logistic regression analysis of predictor variables for sutureless prosthesis receipt and perioperative short-term morbidity was performed with estimated odds ratios (ORs) and 95\% confidence intervals (CIs) for each of the independent variables in the model displayed.

A propensity score, indicating the predicted probability of the patient receiving a sutureless prosthesis, was calculated using a nonparsimonious multiple logistic regression analysis (Appendix E2 and Figure E1) from the logistic equation for each patient. All the preoperative variables listed in Appendix E1 (first 63 on the list) were included in the analysis.

A Cox multivariate proportional hazards regression model, based on 110 preoperative and postoperative variables known to affect survival after AVR, was developed to describe the adjusted mortality risk for various prosthesis groups and to identify independent predictors of follow-up mortality in the studied population. The type of prosthesis variable was entered at the second step of the hazard model, thus showing the hazard ratio (HR) variance over and above the effects of covariates, and adjusted for any baseline differences between the compared groups. HRs with $95 \%$ CIs were 


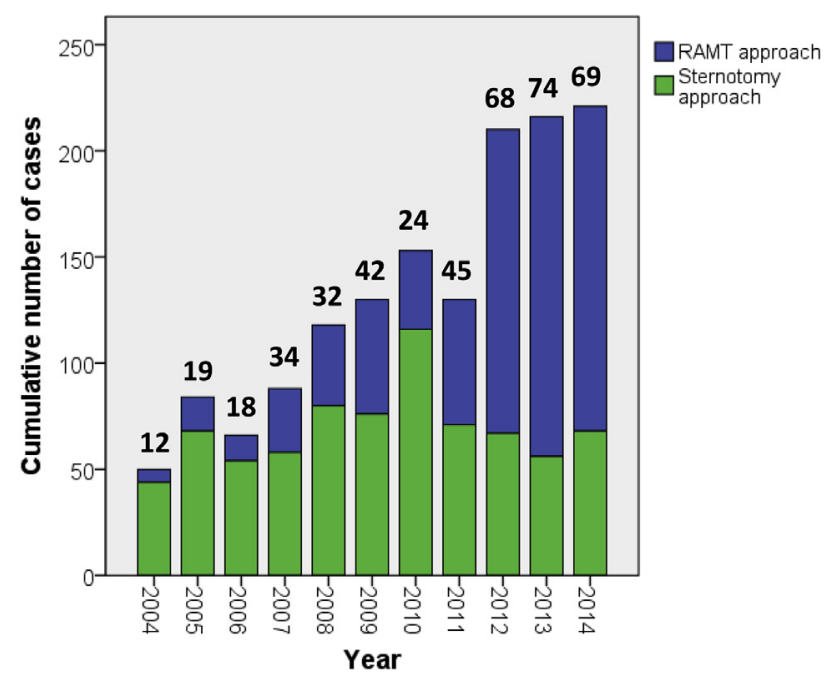

FIGURE 1. Proportion of aortic valve replacement cases performed via RAMT over time, year by year (numbers above the bars indicate $\%$ of the total number of isolated aortic valve replacement operations). Sternotomy approach (green bar) includes both full and partial sternotomy. Cases involving RAMT (blue bar) indicate that since 2011, the overall number of AVR cases has increased, owing to the RAMT component. RAMT, Right anterior minithoracotomy.

calculated for each of the significant risk factors. All reported probability tests are 2-tailed. All data analysis was performed with SPSS statistical software package, version 19.0 (SPSS Inc, Chicago, Ill).

\section{RESULTS}

Within the 10-year study period (2004-2014), 593 patients underwent AVR through RAMT (Figure 1). Baseline demographic and preoperative data of the study population are presented in Table 1. The sample was heterogeneous: for example, $93(15.7 \%)$ patients were aged $\leq 60$ years, and $144(24.3 \%)$ were aged $\geq 80$ years. Compared with the sutured prostheses cohort, the sutureless one was represented by older and sicker patients, with a higher prevalence of tricuspid aortic valve and aortic stenosis, but fewer smokers (Table 1).

The multivariable logistic regression model showed that older age $(P<.0001)$, a higher logistic EuroSCORE (European system for cardiac operative risk evaluation) $(P=.036)$, 4th quartile of logistic EuroSCORE $(P=.002)$, being shorter in height $(P<.0001)$, obesity $(P<.0001)$, tricuspid aortic valve $(P<.0001)$, preoperative atrial fibrillation $(P<.0001)$, cerebrovascular disease $(P=.02)$, aortic valve stenosis $(P<.0001)$, and stenosis with incompetence $(P=.004)$, previous heart surgery $(P=.001)$, and operative priority $(P=.001)$ were independently associated with the sutureless group, whereas first $(P=.0007)$ and second $(P=.03)$ quartiles of age, smoking history $(P=.0004), 3 \mathrm{rd} / 4$ th-grade chronic obstructive pulmonary disease $(P=.02)$, prior cardiac arrhythmia $(P<.0001)$, and III-IV functional class by NYHA $(P<.0001)$ were linked to sutured prosthesis implantation
( $-2 \log$-likelihood $=425.0 ; P=.874$ for lack of fit by Hosmer-Lemeshow test).

Intraoperative characteristics are listed in Table 2. In 50 $(8.4 \%)$ patients, femoral arterial cannulation was used to establish CPB. Nine percent of patients underwent associated procedures, mostly on the mitral valve and for atrial fibrillation; no differences were found $(P=.19)$ between the sutured and sutureless groups. One patient underwent small subaortic ventricular septal defect closure with a direct suture, through enlarged aortotomy.

One hundred thirteen $(19.1 \%)$ patients had a small aortic annulus $(\leq 21 \mathrm{~mm}) ; 89 \%$ of patients received a $21-\mathrm{mm}$, $23-\mathrm{mm}$, or $25-\mathrm{mm}$ prosthesis. Surgery duration was significantly shorter with sutureless prostheses compared with sutured ones: CPB duration was $99 \pm 38$ (median: 90) minutes versus $134 \pm 47$ (median: 126) minutes, $P<.0005$; and aortic crossclamping time was $64 \pm 26$ (median: 57) minutes versus $97 \pm 29$ (median: 89) minutes, $P<.0005$ (overall comparison; Table 3).

Thirteen $(2.2 \%)$ patients needed conversion to full sternotomy, owing to: bleeding ( 5 of $13 ; 38.5 \%$ ); unexpected right pleural cavity adhesions ( 2 of $13 ; 15.4 \%$ ); difficult weaning from CPB and "stone heart syndrome" ( 1 of 13 ; $7.7 \%$ ); technical impossibility of advancing the percutaneous venous cannula ( 2 of $13 ; 15.4 \%$ ); perivalvular leakage ( 2 of $13 ; 15.4 \%$ ) (both cases with sutured prosthesis); and arterial hypotension with hemodynamic instability ( 1 of $13 ; 7.7 \%)$. Hemodynamic instability upon aortic crossclamp removal led to intra-aortic counterpulsation balloon placement in $3(0.51 \%)$ patients. Patient outcomes are presented in Table 4. Surgical mortality (death before discharge) was $6(1.0 \%)$, and 30-day in-hospital mortality was $9(1.5 \%)$. Assisted ventilation time was 9.8 hours (mean), with a median of 6 hours; the 25th to 75th percentiles (interquartile range $[\mathrm{IQR}]$ ) was: 5 to 9 hours. The mean ICU stay was 1.5 days, with a median of 1 day (IQR: 1-1 day). Hospital length of stay averaged 6.6 days, with a median of 6 days (IQR: 6 to 7 days).

Because of having a higher preoperative risk profile, patients in the sutureless group had longer ICU and hospital stay duration, and a higher incidence of permanent pacemaker implantation; however, the assisted mechanical ventilation time was shorter. Mortality did not differ in the 2 groups $(P=.75)$. For 2 postoperative outcome variables of particular interest, a multivariable logistic regression model was developed to assess the role of the type of implanted prosthesis in the occurrence of postoperative events/complications (Table 4, $P$ values with asterisks).

Accordingly, a multivariable logistic regression model showed that a propensity score for a sutureless prosthesis (odds ratio [OR] 9.07; 95\% CI: 2.40-34.24; $P=.001$ ); conversion to median sternotomy (OR 9.35; 95\% CI: $1.76-$ $49.51 ; P=.009)$; complex surgery (OR $3.77 ; 95 \% \mathrm{CI}$ : 
TABLE 1. Baseline preoperative data

\begin{tabular}{|c|c|c|c|c|}
\hline Variable & Overall cohort $(\mathrm{n}=\mathbf{5 9 3})$ & Sutureless subgroup $(\mathrm{n}=\mathbf{3 0 2})$ & Sutured subgroup $(n=291)$ & $P$ value \\
\hline Age (y) & $73.8(66.1-79.4)$ & $77.7(72.4-81.7)$ & $68.3(58.9-76.0)$ & $<.0005$ \\
\hline Gender, female & $283(47.7)$ & $190(62.9)$ & $93(32.1)$ & $<.0005$ \\
\hline Height $(\mathrm{cm})$ & $166(160-173)$ & $163(158-170)$ & $170(164-175)$ & $<.0005$ \\
\hline \multirow[t]{2}{*}{ Weight $(\mathrm{kg})$} & $73.5 \pm 13.8$ & $72.5 \pm 14.0$ & $74.4 \pm 13.6$ & .093 \\
\hline & $74(64-81.5)$ & $72(63-80.3)$ & $74(65-82.3)$ & .066 \\
\hline \multirow[t]{2}{*}{ Body mass index $\left(\mathrm{kg} / \mathrm{m}^{2}\right)$} & $26.4 \pm 4.1$ & $27.0 \pm 4.4$ & $25.8 \pm 3.7$ & $<.0005$ \\
\hline & $26.0(23.8-28.7)$ & $26.8(24.0-29.9)$ & $25.4(23.7-27.8)$ & $<.0005$ \\
\hline Obesity & $114(19.2)$ & $81(26.8)$ & $33(11.4)$ & $<.0005$ \\
\hline Arterial hypertension & $412(69.5)$ & $227(75.2)$ & $185(63.8)$ & .004 \\
\hline Hypercholesterolemia & $348(58.7)$ & $188(62.3)$ & $160(55.2)$ & .080 \\
\hline Diabetes mellitus type II & $124(20.9)$ & $78(25.8)$ & $46(15.9)$ & .003 \\
\hline Smoking history & $183(30.9)$ & $63(20.9)$ & $120(41.4)$ & $<.0005$ \\
\hline Family history of CAD & $178(30.0)$ & $93(30.8)$ & $85(29.3)$ & .69 \\
\hline Extracardiac arterial vascular disease & $93(15.7)$ & $57(18.9)$ & $36(12.4)$ & .031 \\
\hline Cerebral arterial vascular disease & $34(5.7)$ & $28(9.3)$ & $6(2.1)$ & $<.0005$ \\
\hline Previous TIA & $4(0.67)$ & $4(1.3)$ & 0 & .12 \\
\hline Previous stroke & $10(1.7)$ & $6(2.0)$ & $4(1.4)$ & .75 \\
\hline Previous cardiac arrhythmia & $106(17.9)$ & $56(18.5)$ & $50(17.2)$ & .60 \\
\hline Preoperative atrial fibrillation & $46(7.8)$ & $43(14.2)$ & $3(1.0)$ & $<.0005$ \\
\hline Previous pacemaker implantation & $12(2.0)$ & $10(3.3)$ & $2(0.7)$ & .037 \\
\hline Chronic renal failure & $30(5.1)$ & $16(5.3)$ & $14(4.8)$ & .79 \\
\hline Dialysis & $6(1.0)$ & $4(1.3)$ & $2(0.7)$ & .69 \\
\hline Infective endocarditis & $8(1.3)$ & 0 & $8(2.8)$ & .003 \\
\hline Chronic obstructive pulmonary disease & $79(13.3)$ & $46(15.2)$ & $33(11.4)$ & .17 \\
\hline With severe obstruction & $29(4.9)$ & $13(4.3)$ & $16(5.5)$ & .49 \\
\hline Chronic bronchodilator use & $26(4.4)$ & $21(7.0)$ & $5(1.7)$ & .002 \\
\hline $\begin{array}{l}\text { Previous interventional cardiologic } \\
\text { procedure }\end{array}$ & $55(9.3)$ & $36(11.9)$ & $19(6.6)$ & .024 \\
\hline Redo surgery & $9(1.5)$ & $7(2.3)$ & $2(0.7)$ & .18 \\
\hline Previous CABG & $3(0.51)$ & $2(0.7)$ & $1(0.3)$ & $>.99$ \\
\hline Previous valvular surgery & $5(0.84)$ & $5(1.7)$ & 0 & .062 \\
\hline Other cardiac surgery & $3(0.51)$ & $1(0.3)$ & $2(0.7)$ & 62 \\
\hline Previous myocardial infarction & $20(3.4)$ & $13(4.3)$ & $7(2.4)$ & .20 \\
\hline Angina pectoris & $76(12.8)$ & $45(14.9)$ & $31(10.7)$ & .13 \\
\hline Tumor & $11(1.9)$ & $7(2.3)$ & $4(1.4)$ & .40 \\
\hline Preoperative critical status* & $2(0.33)$ & $1(0.3)$ & $1(0.3)$ & $>.99$ \\
\hline Liver cirrhosis & $5(0.84)$ & $3(1.0)$ & $2(0.7)$ & $>.99$ \\
\hline Congestive heart failure & $68(11.5)$ & $40(13.2)$ & $28(9.7)$ & .17 \\
\hline Functional class III or IV by NYHA & $171(28.8)$ & $79(26.2)$ & $92(31.7)$ & .14 \\
\hline Aortic transvalvular gradient $(\mathrm{mm} \mathrm{Hg})$ & $80(62-97)$ & $78(62-94)$ & $81(65-99)$ & .15 \\
\hline Left ventricular ejection fraction $(\%)$ & $60(55-60)$ & $60(55-60)$ & $60(55-60)$ & .82 \\
\hline Left ventricular ejection fraction $\leq 0.5$ & $61(10.3)$ & $31(10.3)$ & $30(10.3)$ & .97 \\
\hline Left ventricular ejection fraction $\leq 0.3$ & $8(1.3)$ & $6(2.0)$ & $2(0.7)$ & .29 \\
\hline Aortic valve pathology & & & & $<.0005$ \\
\hline Stenosis & $452(76.2)$ & $274(90.7)$ & $178(61.2)$ & \\
\hline Mixed lesion & $55(9.3)$ & $22(7.3)$ & $33(11.3)$ & \\
\hline Regurgitation & $86(14.5)$ & $6(2.0)$ & $80(27.5)$ & \\
\hline Bicuspid aortic valve & $127(21.5)$ & $33(10.9)$ & $94(32.3) \dagger$ & $<.0005$ \\
\hline Severe pulmonary hypertension & $28(4.7)$ & $28(9.3)$ & 0 & $<.0005$ \\
\hline Urgency or emergency & $86(14.5)$ & $59(19.5)$ & $27(9.3)$ & $<.0005$ \\
\hline EuroSCORE version I & $6.00(5.00-8.00)$ & $7.00(6.00-9.00)$ & $5.00(3.00-7.00)$ & $<.0005$ \\
\hline Logistic EuroSCORE version I & $5.76(3.28-9.52)$ & $7.9(4.8-11.4)$ & $4.1(2.1-6.3)$ & $<.0005$ \\
\hline
\end{tabular}


TABLE 2. Intraoperative data

\begin{tabular}{|c|c|c|c|c|}
\hline Variable & $\begin{array}{l}\text { Overall cohort } \\
\quad(\mathbf{n}=\mathbf{5 9 3})\end{array}$ & $\begin{array}{l}\text { Sutureless subgroup } \\
\quad(\mathbf{n}=\mathbf{3 0 2})\end{array}$ & $\begin{array}{l}\text { Sutured subgroup } \\
\quad(\mathbf{n}=\mathbf{2 9 1})\end{array}$ & $P$ value \\
\hline Associated procedures & $52(8.8)$ & 22/302 (7.3) & $30 / 291(10.3)$ & .19 \\
\hline Mitral valve procedure & $33 / 52(63.5)$ & $17 / 22(77.3)$ & $16 / 30(53.3)$ & .077 \\
\hline Repair & $21 / 33(63.6)$ & & & \\
\hline Replacement & $12 / 33(36.4)$ & & & \\
\hline Tricuspid valve procedure & $6 / 52(11.5)$ & $2 / 22(9.1)$ & $4 / 30(13.3)$ & $>.99$ \\
\hline Atrial fibrillation ablation & $15 / 52(28.8)$ & 0 & $15 / 30(50.0)$ & $<.0005$ \\
\hline $\begin{array}{l}\text { Others (Morrow ventricular septal myectomy, left atrial appendage } \\
\text { closure, VSD repair, aortic annulus reconstruction) }\end{array}$ & $8 / 52(15.4)$ & $3 / 22(13.6)$ & $5 / 30(16.7)$ & $>.99$ \\
\hline Other noncardiac procedure (quadrantectomy, iliac vein repair) & $4 / 52(7.7)$ & $2 / 22(9.1)$ & $2 / 30(6.7)$ & $>.99$ \\
\hline Implanted prosthesis size $(\mathrm{mm})$ & & & & .002 \\
\hline 19 & $7(1.2)$ & $2(0.7)$ & $5(1.7)$ & \\
\hline 21 & $83(14.0)$ & $38(12.6)$ & $45(15.5)$ & \\
\hline 22 & $2(0.34)$ & 0 & $2(0.7)$ & \\
\hline 23 & $191(32.2)$ & $96(31.8)$ & $96(33.0)$ & \\
\hline 25 & $254(42.8)$ & $149(49.3)$ & $104(35.7)$ & \\
\hline 27 & $53(8.9)$ & $17(5.6)$ & $36(12.3)$ & \\
\hline 29 & $3(0.51)$ & 0 & $3(1.0)$ & \\
\hline Median (interquartile range) of implanted prosthesis size $(\mathrm{mm})$ & $25(23-25)$ & $25(23-25)$ & $23(23-25)$ & .60 \\
\hline Sutureless prosthesis* & $286(48.2)$ & $286(94.7)$ & & \\
\hline Pericardial bioprosthesis $\dagger$ & $245(41.3)$ & & $245(84.2)$ & \\
\hline Porcine bioprosthesis $\ddagger$ & $20(3.4)$ & & $20(6.9)$ & \\
\hline Mechanical prosthesis $\S$ & $18(3.0)$ & & $18(6.2)$ & \\
\hline Mechanical prosthesis $\llbracket$ & $3(0.51)$ & & $3(1.0)$ & \\
\hline Mechanical prosthesis $\|$ & $2(0.34)$ & & $2(0.7)$ & \\
\hline Aortic valve system\# & $10(1.7)$ & $10(3.3)$ & & \\
\hline Sutureless bioprosthesis** & $6(1.0)$ & $6(2.0)$ & & \\
\hline Others & $3(0.51)$ & & $3(1.0)$ & \\
\hline
\end{tabular}

Values are $\mathrm{n}(\%)$, unless otherwise indicated. VSD, Ventricular septal defect. *Perceval S (Sorin, Saluggia, Italy). †Perimount (Carpentier-Edwards, Irvine, Calif). $\ddagger$ Mosaic (Medtronic, Minneapolis, Minn). §(CarboMedics, Austin, Tex). $\uparrow($ St Jude Medical, St Paul, Minn). \|Bicarbon Slimline (Sorin, Saluggia, Italy). \#Edwards Intuity (Edwards Lifesciences, Irvine, Calif). **ATS 3F Enable (Medtronic, Minneapolis, Minn).

$1.16-12.25 ; P=.027)$; artificial ventilation time $>12$ hours (OR 3.61; 95\% CI: 1.37-9.49, $P=.009$ ); and respiratory dysfunction (OR 7.25; 95\% CI: 3.00-17.53; $P<.0005$ ) were independently associated with a $\geq 3$-day ICU stay ( $P=.83$ for goodness of fit by Hosmer-Lemeshow; -2 $\log$-likelihood $=180.4$ ). Likewise, complete postoperative atrioventricular block with necessity of permanent pacemaker implantation was predicted $(P=.72$ for goodness of fit by Hosmer-Lemeshow, -2 log-likelihood = 128.5), by family history of CAD (OR 3.1; 95\% CI: 1.14-8.47; $P=.03$ ); logistic EuroSCORE (OR 1.17; 95\% CI: 1.06$1.29 ; P=.002)$; complex surgery (OR 9.99; 95\% CI: 2.00-49.94; $P=.005$ ); and patient's height (OR 0.922; 95\% CI: 0.865-0.983; $P=.014)$.
In $98(16.5 \%)$ patients, the composite event score was $\geq 1$ (range: $1-5$ ), resulting in a total sum of events equal to 148. By ordinal regression analysis, independent contributors were age quartiles (first age quartile $2.17 ; 95 \% \mathrm{CI}$ : $0.02-4.31 ; P=.047$, and second age quartile $1.52 ; 95 \%$ CI: $0.11-2.93 ; P=.035)$, extracardiac arterial vascular disease $(1.81,95 \%$ CI: $0.44-3.18 ; P=.01)$; previous CABG (13.7, 95\% CI: 9.8-17.5; $P<.0005)$; previous valvular surgery $(11.4 ; 95 \% \mathrm{CI}: 8.5-14.4 ; P<.0005)$, and $\mathrm{CPB}$ time (0.024; 95\% CI: $0.012-0.037 ; P<.0005)$, whereas NYHA classification had only marginal significance $(-1.98 ; 95 \%$ CI: -4.06 to $0.11 ; P=.06$ for NYHA class I).

One $(0.17 \%)$ patient, a woman aged 83 years, experienced acute aortic dissection on the third postoperative

TABLE 3. Operative times: overall data and comparison among subgroups

\begin{tabular}{lcccc}
\hline & $\begin{array}{c}\text { Overall study } \\
\text { Vopulation }(\mathbf{n}=\mathbf{5 9 3})\end{array}$ & $\begin{array}{c}\text { Isolated AVR } \\
(\mathbf{n}=\mathbf{5 4 1})\end{array}$ & $\begin{array}{c}\text { Isolated AVR with sutured } \\
\text { prostheses }(\mathbf{n}=\mathbf{2 5 8})\end{array}$ & $\begin{array}{c}\text { Isolated AVR with } \\
\text { sutureless prostheses } \\
(\mathbf{n}=\mathbf{2 8 3})\end{array}$ \\
\hline CPB time $(\mathrm{min})$ & $107(86-135)$ & $104(84-129)$ & $121(103.5-147.5)$ & $88(77-107)$ \\
ACC time $(\mathrm{min})$ & $74(55-96)$ & $71(54-92)$ & $87(75-109)$ & $55(47-68)$ \\
\hline For isolated AVR, $P<.0005$ (for both $\mathrm{CPB}$ and ACC times), compared with associated procedures (eg, AVR and/or mitral procedure and/or Morrow septal myectomy). For
\end{tabular}
sutureless versus sutured comparison, $P<.0005$ (for both $\mathrm{CPB}$ and ACC times). $A V R$, Aortic valve replacement; $C P B$, cardiopulmonary bypass; $A C C$, aortic cross clamp. 
TABLE 4. Overall patient outcomes

\begin{tabular}{|c|c|c|c|c|}
\hline Variable & $\begin{array}{l}\text { Overall cohort } \\
\quad(\mathbf{n}=\mathbf{5 9 3})\end{array}$ & $\begin{array}{l}\text { Sutureless subgroup } \\
\quad(\mathbf{n}=\mathbf{3 0 2})\end{array}$ & Sutured subgroup $(\mathrm{n}=291)$ & $P$ value \\
\hline ICU length of stay (d) & $1(1-1)$ & $1(1-1)(70$ cases $>$ median $)$ & $1(1-1)(44$ cases $>$ median $)$ & .011 \\
\hline Prolonged ICU stay & $53(8.9)$ & $34(11.3)$ & $19(6.6)$ & $.046^{*}$ \\
\hline Assisted ventilation time $(\mathrm{h})$ & $6(5-9)$ & $6(4-8)$ & $7(5-9)$ & .004 \\
\hline Prolonged ventilation support & $22(3.7)$ & $9(3.0)$ & $13(4.5)$ & .33 \\
\hline Hospital length of stay (d) & $6(6-7)$ & $6(6-8)$ & $6(6-7)$ & .027 \\
\hline In-hospital mortality & $9(1.5)$ & $4(1.3)$ & $5(1.7)$ & .75 \\
\hline Reopening for bleeding or cardiac tamponade & $30(5.1)$ & $17(5.6)$ & $13(4.5)$ & .53 \\
\hline Perioperative acute myocardial infarction & $9(1.5)$ & $3(1.0)$ & $6(2.1)$ & .33 \\
\hline Infective complications & $19(3.2)$ & $9(3.0)$ & $10(3.4)$ & .75 \\
\hline Sepsis & $1(0.17)$ & $1(0.3)$ & 0 & $>.99$ \\
\hline Wound dehiscence or infection & $3(0.51)$ & $1(0.3)$ & $2(0.7)$ & 62 \\
\hline Stroke & $10(1.7)$ & $6(2.0)$ & $4(1.4)$ & .75 \\
\hline Transient ischemic attack & $6(1.0)$ & $3(1.0)$ & $3(1.0)$ & $>.99$ \\
\hline Pulmonary complications/respiratory dysfunction & $65(11.0)$ & $36(11.9)$ & $29(10.0)$ & .46 \\
\hline \multirow[t]{2}{*}{ Pneumonia } & $7(1.2)$ & $1(0.3)$ & $6(2.1)$ & .064 \\
\hline & $7 / 65(10.8)$ & $1 / 36(2.8)$ & $6 / 29(20.7)$ & .039 \\
\hline Pleural effusion requiring puncture & $16(2.7)$ & $11(3.6)$ & $5(1.7)$ & .15 \\
\hline Perioperative acute kidney injury & $23(3.9)$ & $13(4.3)$ & $10(3.4)$ & .59 \\
\hline New-onset CVVH/hemofiltration support & $2(0.34)$ & $1(0.3)$ & $1(0.3)$ & $>.99$ \\
\hline Complete AV block; requested PM implant & $19(3.2)$ & $14(4.6)$ & $5(1.7)$ & $.045^{*}$ \\
\hline New-onset atrial fibrillation or flutter & $151(25.5)$ & $78(25.8)$ & $73(25.2)$ & .86 \\
\hline Gastrointestinal complications & $8(1.3)$ & $7(2.3)$ & $1(0.3)$ & .069 \\
\hline
\end{tabular}

Values are n (\%), or median (range), unless otherwise indicated. $I C U$, Intensive care unit; $C V V H$, continuous veno-venous hemofiltration/hemodialysis; $A V$, atrioventricular; $P M$, pacemaker. *Statistically insignificant $P$ values for the type of valvular prosthesis variable in multivariable model predicting this event/complication.

day and underwent reoperation to replace the aortic root and ascending aorta.

Nine $(1.5 \%)$ patients were lost to follow-up. At 31.5 months, the mean follow-up time (median: 20.1; IQR: 11.1-51.5 months), with 1531 accumulated patient-years, $94.8 \%$ (562 of 593) survival was observed. Patients in the sutureless group had a shorter follow-up time: 12.6 (5.419.0) months versus 51.5 (26.5-66.6) months, $P<.0005$.

Twenty-two late deaths have been observed during follow-up, 3 of them due to cardiac pathology. Eleven (11 of $584 ; 1.9 \%$ ) failed prostheses necessitated reoperation: 2 patients from the sutureless subgroup, and 5 patients from the conventional subgroup have undergone reoperation for infective endocarditis, and 4 patients from the conventional subgroup underwent redo AVR for a degenerated bioprosthesis. Overall actuarial survival by Kaplan-Meier at $1,3,5$, and 8 years was $96.5 \% \pm 1 \%, 94.1 \% \pm 1 \%$, $93.3 \% \pm 2 \%$, and $93.3 \% \pm 2 \%$, respectively (Figure 2 ). Actuarial survival was similar in the sutureless versus conventional prostheses groups ( $P=.11$ by log-rank), but it was limited with a shorter follow-up time in the sutureless group (Figure 3). Actuarial freedom from reoperation at 5 years was $97.2 \% \pm 2 \%$.

Late survival by a Cox multivariable proportional hazards regression model was associated with 3 independent predictors: preoperative cardiac arrhythmia (hazard ratio [HR] 2.58; 95\% CI: 1.06-6.28; $P=.036)$, logistic EuroSCORE (HR 1.067; 95\% CI: $1.007-1.131 ; P=.029$ ), and artificial ventilation time (HR 1.014; 95\% CI: 1.006 $1.022, P<.0005)$, but it was not affected by the sutureless/sutured variable (HR 1.02; 95\% CI: 0.33-3.14, $P=.97$ ) (Figure 4).

\section{DISCUSSION}

Many centers have reported their midterm and remote results after mitral valve surgery via right anterior minithoracotomy (RAMT), but only a few have published articles

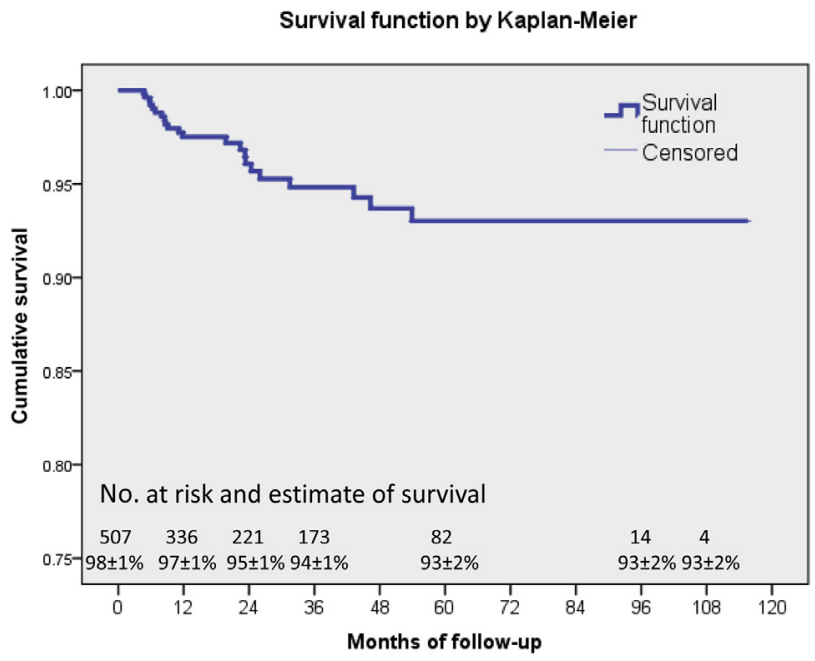

FIGURE 2. Overall survival function by Kaplan-Meier test. 


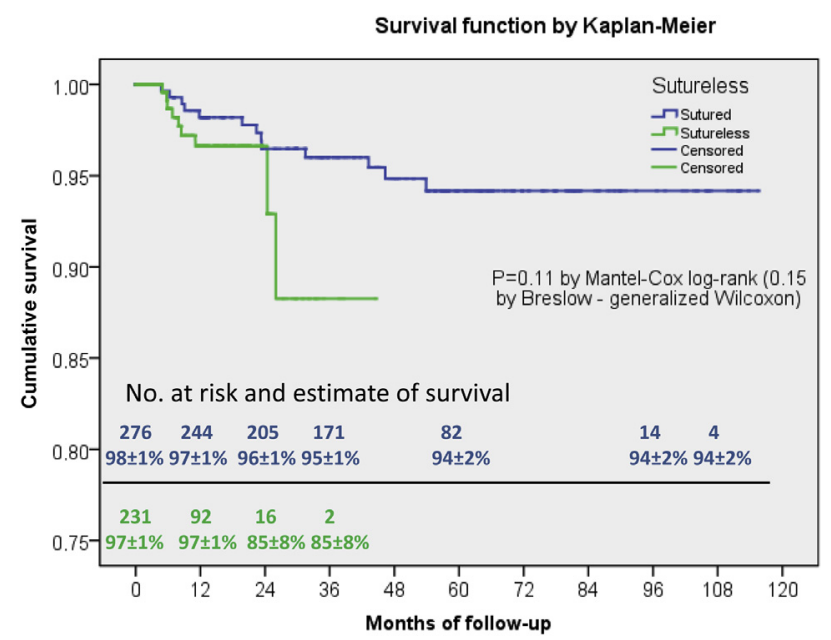

FIGURE 3. Comparison of survival by Kaplan-Meier between sutured and sutureless cohort. Green line and green numbers refer to sutureless prostheses; blue line and blue numbers refer to sutured prostheses. Shorter follow-up in the sutureless group, with information lacking over 24 months, is evident by "block" shape of green line.

regarding AVR..$^{9-12}$ To our knowledge, this is the largest single-center experience to date on the minimally invasive AVR via RAMT. Our data, along with earlier reports, confirm that minimally invasive AVR via RAMT is safe and reproducible. It is associated with low perioperative mortality and morbidity.

We began our clinical experience with RAMT AVR in mid-2004, based on the minimally invasive mitral valve surgery setting. Physiologic antegrade perfusion should be preferred, to avoid cerebral and splanchnic embolic complications. Surgical exposure of femoral vessels is additionally associated with groin complications. ${ }^{4}$ The standardized

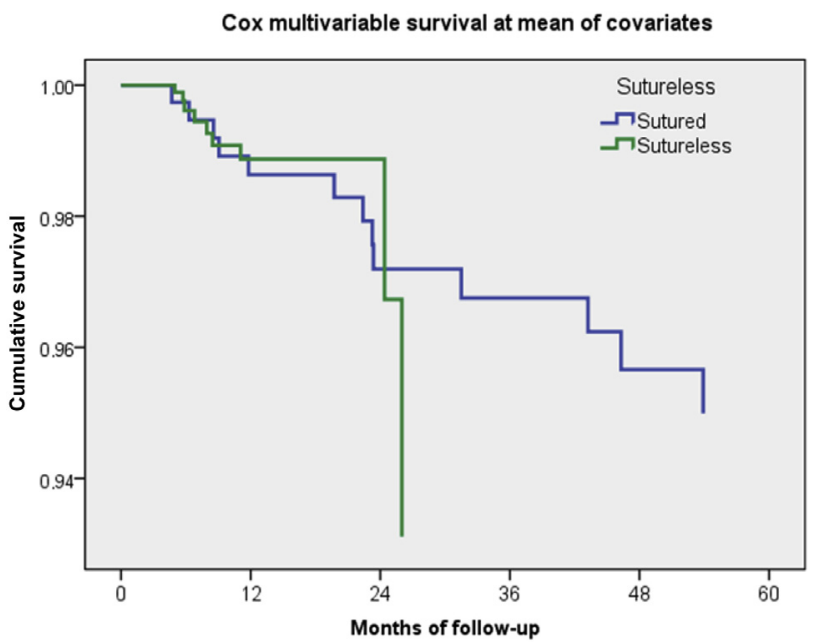

FIGURE 4. Follow-up survival by Cox multivariable proportional hazards regression model at mean of covariates, stratified by the type of aortic valve prosthesis (hazard ratio 1.02 ; $95 \% \mathrm{CI}$ : $0.33-3.14 ; P=.97$ ). Shorter follow-up in the sutureless group, with information lacking over 24 months, is evident by "block" shape of green line. surgical technique used by all attending surgeons in our department may be the reason for success. ${ }^{24}$ Teamwork and patient care continuity, from operating room to ICU and ordinary ward, are of essential importance. Figure 1 shows the substantial increase in the number of patients operated on with RAMT since 2011, which is the year that clinical application of sutureless technology began to increase in our institution.

Since our very early experience with RAMT, through interim analysis, we have been gaining clear confidence that reduced surgical trauma, compared with sternotomy, is transformed into improved perioperative outcomes. Subsequently, we tried to recruit more patients who were operable via RAMT; however, patients' individual anatomy and CT selection criteria created natural limits on the number. Nonetheless, increased experience with RAMT allowed us to make definite progress, and between 2005 and 2010, we steadily increased the number of RAMT procedures (Figure 1).

The population in Italy is rapidly gaining a larger proportion of elderly people, and this trend is common in the postindustrial world. This increasing proportion consists of people who are older and sicker, are affected by the substantial impact of associated comorbidities and frailty, and need a biological prosthesis rather than a mechanical one. These factors, along with the necessity of maintaining at least the same outcome rate after AVR, despite a worse baseline risk profile, were the impetus for entering the sutureless era at our institution.

Sutureless prostheses came to cover a vast slice of patients, who were eligible, on the one hand, for biological prosthesis implantation; on the other hand, they requested less-invasive technique implementation because of their poorer baseline conditions. Sutureless aortic valve prostheses may enhance the minimal invasiveness of surgical incision, especially with right minithoracotomy, in which the chest bone structure is not divided at any point to reach pericardium. The complementary character of a sutureless prosthesis implanted with a really minimally invasive technique-RAMT_-forsaged an important paradigm shift. Today, our first-line option for an average patient with degenerative aortic valve stenosis is sutureless AVR via right minithoracotomy, unless it is contraindicated by some exclusion criteria (eg, regarding right chest abnormality or venous anatomy). We feel that sutureless technology, facilitating the surgical technique and rendering it step-bystep standardizable, could contribute to a wider adoption of minimally invasive AVR.

In our case series, a single patient underwent stentless AVR in the RAMT approach. In contrast to Sansone and colleagues, ${ }^{25}$ we were not satisfied with aortic root exposure with a stentless prosthesis inside, with the limited space to work and reduced angle of instrument movement that resulted in cumbersome suture placement. Thus, we thereafter 
reserved a partial sternotomy for minimally invasive stentless AVR, in which a lower border of surgical incision of the thoracic wall allows better amplitude of surgical maneuvers. However, even Sansone and colleagues ${ }^{25}$ had to enlarge the initial incision to transverse sternotomy in 2 of 7 patients.

Minimally invasive AVR has been associated by most authors with better perioperative outcomes, ${ }^{5,6,8,10,13}$ but constantly longer operative times, compared to full sternotomy. Extremely variable perfusion times with RAMT AVR have been reported. ${ }^{4,8,18}$ Our data, observed with conventional prostheses, are in line with previous reports, but they are much improved with use of sutureless prostheses.

As in previous reports, ${ }^{20,21}$ we showed that sutureless prostheses significantly reduce operative times and provide at least comparable clinical outcomes. Indeed, direct univariate comparison favored the sutured group with regard to early postoperative outcomes (Table 4), but more-robust multivariable regression model analysis did not indicate that conventional prostheses had better general performance. Several confounders (eg, age, female gender, obesity), revealed by multivariable logistic regression analysis to be independently associated with the sutureless group, were underlying factors.

Although it was projected as a tool for isolated AVR, RAMT does not preclude use of various associated procedures, and even triple-valve surgery is possible in selected patients. Five patients underwent triple-valve surgery in this series, ${ }^{26}$ and we agree with Elmahdy et al. ${ }^{27}$ on the technical feasibility of this approach. In addition, some patients undergoing a reoperation can be treated for AVR via RAMT, when preoperative computed tomography evaluation does not exclude this surgical approach.

The issue of limited space during AVR via RAMT is handled easily with the choice of sutureless prosthesis. However, our experience shows that not every type of sutureless aortic valve prosthesis can by implanted through RAMT. The most useful and effective concept in such circumstances seems to be a collapsible device. A collapsible profile of the prosthesis enhances visualization in a reduced surgical exposure site and allows a surgeon to secure its correct position under direct visual control before deployment. Wider use of sutureless prostheses may increase adoption of minimally invasive AVR, especially with RAMT.

Multivariable logistic analysis failed to indicate independent predictors of in-hospital mortality in the present study, whereas Sharony and colleagues ${ }^{11}$ linked hospital mortality to congestive heart failure, urgent surgery, depressed left ventricular function, and age. We may suppose that in our population, hospital mortality was rare (only 9 patients) and was casually associated with various factors, probably not those considered in the database, that precluded confident statistical identification of them. However, 3 independent predictors of late mortality have been demonstrated by Cox multivariable regression analysis.
Permanent pacemaker implantation after sutureless AVR is still a topic of intense debate. In the present study, complete postoperative atrioventricular block was linked to a family history of CAD, a higher logistic EuroSCORE, and patient's height, and not to sutureless treatment, even though the latter seemed to affect atrioventricular block incidence, as assessed by univariate analysis. This empirical analysis, however, lacked more-specific data (eg, degree of aortic annulus decalcification, preoperative electrocardiogram abnormalities, and electrophysiologic evaluation of the block), which might have more-precise prognostic significance. Experience on the subject is growing, and we will continue our research.

\section{Limitations}

Several limitations of our study have to be noted. It is based on a retrospective analysis of our large, institutional, observational, prospectively collected database; thus, it reflects single-center experience only and carries all the limits that a retrospective analysis design implies. Our internal institutional electronic database contains EuroSCORE-Ibased records, instead of the newly introduced and more efficient EuroSCORE II. In addition, this study reflects our initial experience with the RAMT approach and encompasses the "learning curve."

Given that ours is a retrospective study without a control group, unadjusted biases may have confounded our findings. We might have been unable to account for the influence of residual unmeasured factors that could affect adverse outcomes. On the other hand, this experience with "all comers" shows real-world data.

We fully recognize that our case series is extremely heterogeneous, and comprises various clinical scenarios. An extremely heterogeneous study population may account for the skewness of continuous data, and the abnormal distribution of dichotomous data. However, with a multivariable regression model-based propensity score adjustment, we attempted to smooth the differences between the 2 main groups-sutured and sutureless. For outcome incidence, which differed in the 2 groups, specific multivariable regression models were created to adjust for confounders. Despite a Cox regression model that identified several independent predictors of late mortality, this analysis is limited by the low number of events that could have affected the result, as shown by a wide CI.

Further, statistics cited earlier were based on a median follow-up time of approximately 1 year for sutureless prostheses, accounting for clearly unequal follow-up duration, compared with conventional prostheses. Thus, a longer duration is needed to confirm durability and hemodynamic performance of the sutureless prostheses. We have utilized all-cause mortality data, although they were reliably obtained from our database, rather than the more-specific cardiac-related mortalities, and we did not address the 
relative incidence of nonfatal cardiac-related events. At the same time, comprehensive analysis was performed on the whole study population, without any selection; thus, it is a real-world study on the largest, to our knowledge, singlecenter experience to date with RAMT AVR.

\section{CONCLUSIONS}

Minimally invasive AVR is a safe procedure, with low perioperative morbidity, and low rates of reoperation and death at late follow-up. Excellent outcomes can be achieved with minimally invasive AVR through RAMT. Sutureless prostheses facilitate minimally invasive AVR and are associated with reduced operative times.

\section{Conflict of Interest Statement}

Dr Glauber and Dr Solinas have a commercial/financial relationship with Sorin Group. The other authors have nothing to disclose regarding commercial support.

\section{References}

1. Rao PN, Kumar AS. Aortic valve replacement through right thoracotomy. Tex Heart Inst J. 1993;20:307-8.

2. Cosgrove DM, Sabik JF. Minimally invasive approach for aortic valve operations. Ann Thorac Surg. 1996;62:596-7.

3. von Segesser LK, Westaby S, Pomar J, Loisance D, Groscurth P, Turina M. Less invasive aortic valve surgery: rationale and technique. Eur J Cardiothorac Surg. 1999; 15:781-5.

4. Ruttmann E, Gilhofer TS, Ulmer H, Chevtchik O, Kocher A, Schistek R, et al. Propensity score-matched analysis of aortic valve replacement by mini-thoracotomy. J Heart Valve Dis. 2010;19:606-14.

5. Tabata M, Umakanthan R, Cohn LH, Bolman RM III, Shekar PS, Chen FY, et al. Early and late outcomes of 1000 minimally invasive aortic valve operations. Eur J Cardiothorac Surg. 2008;33:537-41.

6. Brown ML, McKellar SH, Sundt TM, Schaff HV. Ministernotomy versus conventional sternotomy for aortic valve replacement: a systematic review and meta-analysis. J Thorac Cardiovasc Surg. 2009;137:670-9.

7. Korach A, Shemin RJ, Hunter CT, Bao Y, Shapira OM. Minimally invasive versus conventional aortic valve replacement: a 10-year experience. J Cardiovasc Surg (Torino). 2010;51:417-21.

8. Glower DD, Desai BS, Hughes GC, Milano CA, Gaca JG. Aortic valve replacement via right minithoracotomy versus median sternotomy: a propensity score analysis. Innovations (Phila). 2014;9:75-81.

9. Miceli A, Murzi M, Gilmanov D, Fuga’ R, Ferrarini M, Solinas M, et al. Minimally invasive aortic valve replacement using right minithoracotomy is associated with better outcomes than ministernotomy. J Thorac Cardiovasc Surg. 2014;148:133-7.

10. Sharony R, Grossi EA, Saunders PC, Schwartz CF, Ribakove GH, Baumann FG, et al. Propensity score analysis of a six-year experience with minimally invasive isolated aortic valve replacement. J Heart Valve Dis. 2004;13:887-93.
11. Sharony R, Grossi EA, Saunders PC, Schwartz CF, Ribakove GH, Culliford AT, et al. Minimally invasive aortic valve surgery in the elderly: a case-control study. Circulation 2003;108 Suppl 1:II43-7.

12. Glauber M, Miceli A, Gilmanov D, Ferrarini M, Bevilacqua S, Farneti PA, et al. Right anterior minithoracotomy versus conventional aortic valve replacement: a propensity score matched study. J Thorac Cardiovasc Surg. 2013;145:1222-6.

13. Ghanta RK, Lapar DJ, Kern JA, Kron IL, Speir AM, Fonner E Jr, et al. Minimally invasive aortic valve replacement provides equivalent outcomes at reduced cost compared with conventional aortic valve replacement: a real-world multiinstitutional analysis. J Thorac Cardiovasc Surg. 2015;149:1060-5.

14. Murtuza B, Pepper JR, Stanbridge RD, Jones C, Rao C, Darzi A, et al. Minimal access aortic valve replacement: Is it worth it? Ann Thorac Surg. 2008;85: 1121-31.

15. Bakir I, Casselman FP, Wellens F, Jeanmart H, De Geest R, Degrieck I, et al. Minimally invasive versus standard approach aortic valve replacement: a study in 506 patients. Ann Thorac Surg. 2006;81:1599-604.

16. Furukawa N, Kuss O, Aboud A, Schönbrodt M, Renner A, Hakim Meibodi K, et al. Ministernotomy versus conventional sternotomy for aortic valve replacement: matched propensity score analysis of 808 patients. Eur J Cardiothorac Surg. 2014;46:221-7.

17. Gosev I, Kaneko T, McGurk S, McClure SR, Maloney A, Cohn LH. A 16-year experience in minimally invasive aortic valve replacement: context for the changing management of aortic valve disease. Innovations (Phila). 2014;9:104-10.

18. Brinkman WT, Hoffman W, Dewey TM, Culica D, Prince SL, Herbert MA, et al. Aortic valve replacement surgery: comparison of outcomes in matched sternotomy and PORT ACCESS groups. Ann Thorac Surg. 2010;90:131-5.

19. Detter C, Deuse T, Boehm DH, Reichenspurner H, Reichart B. Midterm results and quality of life after minimally invasive vs. conventional aortic valve replacement. Thorac Cardiovasc Surg. 2002;50:337-41.

20. Shrestha M, Timm R, Hoeffler K, Koigeldiyev N, Khaladj N, Hagl C, et al. Minimally invasive aortic valve replacement with self-anchoring Perceval valve. $J$ Heart Valve Dis. 2013;22:230-5.

21. Santarpino G, Pfeiffer S, Concistrè G, Grossmann I, Hinzmann M, Fischlein T. The Perceval S aortic valve has the potential of shortening surgical time: Does it also result in improved outcome? Ann Thorac Surg. 2013;96:77-82.

22. Glauber M, Farneti A, Solinas M, Karimov J. Aortic valve replacement through a right minithoracotomy. Multimed Man Cardiothorac Surg. 2006 (1110):mmcts.2005.001826. http://dx.doi.org/10.1510/mmcts.2005.001826.

23. Glauber M, Miceli A, Bevilacqua S, Farneti PA. Minimally invasive aortic valve replacement via right anterior minithoracotomy: early outcomes and midterm follow-up. J Thoracic Cardiovasc Surg. 2011;142:1577-9.

24. Malaisrie SC, Barnhart GR, Farivar RS, Mehall J, Hummel B, Rodriguez E, et al. Current era minimally invasive aortic valve replacement: techniques and practice. J Thorac Cardiovasc Surg. 2014;147:6-14.

25. Sansone F, Zingarelli E, Punta G, Flocco R, Dato GM, Forsennati PG, et al. Aortic valve replacement using a stentless bioprosthesis through right minithoracotomy: an initial experience. Heart Lung Circ. 2011;20:543-6.

26. Lio A, Murzi M, Solinas M, Glauber M. Minimally invasive triple valve surgery through a right minithoracotomy. J Thorac Cardiovasc Surg. 2014;148:2424-7.

27. Elmahdy HM, Nascimento FO, Santana O, Lamelas J. Outcomes of minimally invasive triple valve surgery performed via a right anterior thoracotomy approach. J Heart Valve Dis. 2013;22:735-9.

Key Words: aortic valve replacement, minimally invasive aortic valve replacement, right anterior minithoracotomy, sutureless aortic valve prosthesis 
APPENDIX E1. List of variables used for multivariable tests

1. Age*

2. Quartile of age

3. Height

4. Weight

5. Body mass index

6. Obesity

7. Smoking history

8. Hypercholesterolemia

9. Diabetes mellitus

10. Family history of coronary artery disease

11. Preoperative cardiac arrhythmia history

12. Atrial fibrillation history

13. Arterial hypertension

14. Preoperative glycemia

15. Chronic renal insufficiency (anamnestic data)

16. Preoperative serum creatinine $(\mathrm{mg} / \mathrm{dL})$

17. Preoperative dialysis

18. Extracardiac arterial vascular disease

19. Cerebrovascular disease

20. Previous transient ischemic attack

21. Previous stroke

22. Residual neurologic dysfunction

23. Acute stroke ( $<30 \mathrm{~d}$ from onset)

24. Carotid artery echo color Doppler abnormality

25. Infective endocarditis

26. Chronic lung disease

27. Chronic obstructive pulmonary disease

28. Grade 3-4 chronic bronchial obstruction

29. Chronic bronchodilator use

30. Prior percutaneous vascular procedure

31. Prior cardiovascular surgery

32. Prior coronary artery bypass grafting

33. Prior heart valve surgery

34. Prior other cardiac surgery

35. Acute myocardial infarction

36. Remoteness of acute myocardial infarction

37. Angina pectoris

38. Instable angina pectoris

39. Active neoplastic process

40. Critical preoperative status

41. Prior pacemaker implantation

42. Intravenous antibiotics

43. Intravenous nitrates

44. Liver failure

45. Congestive heart failure

46. Cardiogenic shock

47. NYHA functional class

48. Functional class III or IV by NYHA

49. Left ventricular ejection fraction* $(\%)$

50. Coronary artery disease

51. Left common trunk lesion

52. Number of diseased coronary arteries

53. Transvalvular aortic gradient (echocardiography measurement) $(\mathrm{mm} \mathrm{Hg})$
APPENDIX E1. Continued

54. Aortic valve pathology (stenosis, stenosis and incompetence, pure incompetence)

55. Bicuspid aortic valve

56. Mitral valve disease

57. Tricuspid valve disease

58. Pulmonary hypertension

59. Additive EuroSCORE I

60. Logistic EuroSCORE I

61. Quartile of logistic EuroSCORE I

62. Surgery priority

63. Isolated aortic valve replacement

64. Mitral valve surgery

65. Tricuspid valve surgery

66. Atrial fibrillation ablation

67. Other surgical heart procedures

68. Type of implanted prosthesis (sutureless/conventional; biological/mechanical)

69. Commercial (trade) name of the implanted prosthesis

70. Size of the implanted prosthesis $\dagger(\mathrm{mm})$

71. Conversion to full sternotomy

72. Cardioplegia type

73. $\mathrm{CPB}$ duration* (min)

74. Aortic crossclamp duration* (min)

75. Intra-aortic balloon pump use

76. Arterial cannulation site

77. Venous cannulation site

78. Assisted mechanical ventilation duration* (h)

79. Intensive care unit stay* (d)

80. Revision for bleeding or tamponade

81. Revision for any reason

82. Perioperative myocardial infarction

83. Wound dehiscence or infection

84. Groin cannulation site complications

85. Urinary tract infection

86. Postoperative sepsis

87. Infective complications

88. Confusion/delirium postoperatively

89. Postoperative transient ischemic attack

90. Postoperative stroke

91. Pulmonary distress

92. Pulmonary embolism

93. Pneumonia

94. Pleural effusion requiring drainage

95. Acute kidney injury

96. New-onset renal replacement therapy

97. Acute limb ischemia

98. Atrioventricular block requiring permanent pacemaker implantation

99. Postoperative cardiac arrest

100. Pericarditis

101. Relevant pericardial effusion treated conservatively

102. Gastrointestinal complications

103. Multiorgan failure

104. Postoperative new-onset atrial fibrillation 


\section{APPENDIX E1. Continued}

105. Acute postoperative aortic dissection

106. Major cardiac arrhythmia

107. Acute liver failure

108. Composite event score

109. Discharge destination (home, rehabilitation facility, other hospital)

110. Hospital length of stay*

NYHA, New York Heart Association; EuroSCORE, European system for cardiac operative risk evaluation. *Variable tested as continuous, ordinal, and binary. $†$ Variable tested as ordinal.

\section{APPENDIX E2.}

Propensity-score model for sutureless prosthesis implantation: Receiver operating characteristic curve and model fit by $\mathrm{C}$-statistic and Hosmer-Lemeshow criteria.

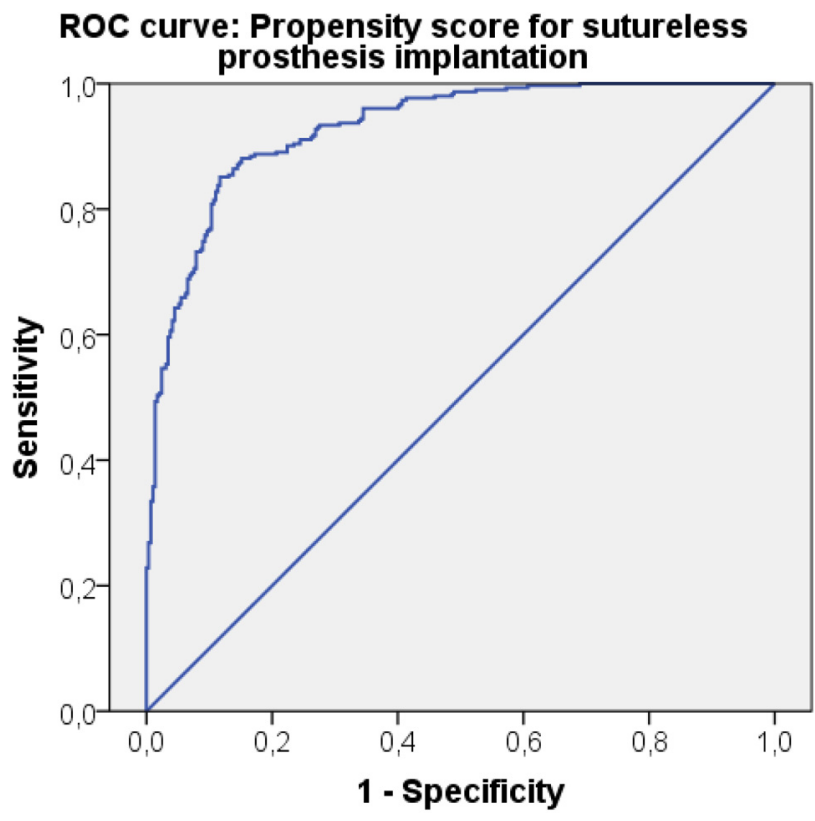

FIGURE E1. Area under the curve $=0.929 ; \mathrm{SE}=0.010 .95 \%$ CI: 0.909 0.948. Overall model fit by Hosmer-Lemeshow: $-2 \log$-likelihood $=398.4$; $\chi^{2}$ statistic $=6.84 ; P=.554 . R O C$, Receiver operating characteristic. 